Mots. Les langages du politique

$88 \mid 2008$

Discours politique, discours expert

\title{
L'expertise syndicale européenne. L'imbrication des lexiques de l'expertise et du discours militant chez les syndicalistes européens
}

Anne-Catherine Wagner

\section{(2) OpenEdition}

Journals

Édition électronique

URL : https://journals.openedition.org/mots/18013

DOI : $10.4000 /$ mots. 18013

ISSN : 1960-6001

Éditeur

ENS Éditions

\section{Édition imprimée}

Date de publication : 1 novembre 2008

Pagination : 53-65

ISBN : 978-2-84788-147-9

ISSN : 0243-6450

\section{Référence électronique}

Anne-Catherine Wagner, «L'expertise syndicale européenne. L'imbrication des lexiques de l'expertise et du discours militant chez les syndicalistes européens ", Mots. Les langages du politique [En ligne], 88 | 2008, mis en ligne le 01 novembre 2010, consulté le 23 avril 2022. URL : http:// journals.openedition.org/mots/18013; DOI : https://doi.org/10.4000/mots.18013 


\section{L'expertise syndicale européenne. L'imbrication des lexiques de l'expertise et du discours militant chez les syndicalistes européens}

La Confédération européenne des syndicats (CES), instituée en 1973 pour représenter et promouvoir les intérêts des travailleurs auprès des institutions européennes, rassemble aujourd'hui à Bruxelles la quasi-totalité des syndicats européens. L'intégration relativement réussie dans l'univers institutionnel européen est à la source de critiques adressées à ce syndicalisme dont le mode de fonctionnement diplomatico-administratif semble bien éloigné des logiques militantes (Pernot, 1998). "Partenaire social», statutairement consulté avant toute proposition dans le domaine social, inscrite dans un « dialogue social » avec le patronat européen, la CES est conduite à développer une expertise qui se construit en réponse aux expertises produites par d'autres institutions européennes, et notamment par la Commission européenne. Corinne Gobin a analysé l'intégration progressive du vocabulaire patronal et du vocabulaire administratif de la Commission au détriment du vocabulaire plus spécifiquement syndical. En comparant les résolutions des congrès successifs de la CES, elle met en évidence l'européanisation du lexique (surtout à partir du congrès de 1991 qui marque le renforcement du rôle institutionnel de la CES). La CES utilise de plus en plus d'expressions issues des discours des autorités communautaires: partenaire (social), intégration (européenne), directive, cohésion, dialogue, normes, contrats; les termes de compétitivité, de flexibilité apparaissent au milieu des années quatre-vingt-dix, alors que le vocabulaire de la lutte, de la revendication et de l'action est peu présent (Gobin, 1993; 2003).

La question que nous voudrions aborder ici est celle de l'effet de ces évolutions sur la manière dont les syndicalistes engagés dans un travail de représentation au niveau européen se représentent leurs propres activités. L’insertion dans l'univers bien particulier des institutions européennes impose de développer des pratiques et des discours spécifiques, qui peuvent sembler éloignés de l'ethos du syndicaliste, tel qu'il s'est historiquement constitué en France. Les syndicalistes français chargés des questions européennes se définissent souvent comme des militants, devenus permanents au terme d'un

Université Paris 1, Centre de sociologie européenne, acwagner@univ-paris1.fr 
parcours dans l'appareil syndical. Dans quelle mesure deviennent-ils de nouveaux professionnels de l'Europe, adoptant un discours expert où sont absents ou minorés les éléments de conflits politiques? Le terme expertise n'est pas en lui-même exempt d'ambigüité: quel sens revêt-il quand il est employé par des syndicalistes? Comment s'articulent le point de vue de l'expert et le point de vue militant dans les discours des acteurs syndicaux européens?

L'objectifest donc de tester la tension lexicale que Corinne Gobin a dégagée dans l'analyse diachronique des résolutions de congrès sur un tout autre corpus: des entretiens semi-directifs, oraux et donc plus informels, menés auprès de syndicalistes spécialistes des questions européennes, concentrés sur une période relativement courte, entre 2000 et 2004 (Wagner, 2005).

\section{L’analyse lexicale des entretiens}

Les personnes interviewées ont en commun de consacrer l'essentiel de leur temps syndical aux activités européennes. On a cherché à diversifier les structures syndicales représentées, en interrogeant des syndicalistes impliqués dans celles-ci à des niveaux différents. Une partie d'entre eux travaille directement dans une institution européenne à Bruxelles (la CES, son institut de recherche syndicale, une fédération syndicale européenne). Certains sont chargés des questions européennes dans une des grandes confédérations françaises (la CGT, la CFDT ou la CGT-FOํ) ou dans une fédération de branche d'une de ces centrales: ils ont alors pour tâche de représenter leur organisation dans les groupes de travail ou dans les instances syndicales européennes, et plus généralement de faire le lien entre les niveaux européen et national. Enfin, le corpus comprend des entretiens avec des militants d'entreprises qui découvrent les instances européennes grâce à l'institution, par une directive de 1994, de comités d'entreprises européens dans les entreprises multinationales. La plupart des interviewés sont français (à l'exception de 5 des 8 syndicalistes interviewés à Bruxelles qui sont belges, portugais, grec, allemand).

Les entretiens, d'une durée variant d'une à trois heures, avaient pour objectif d'enregistrer des récits de pratiques syndicales. La première partie de l'entretien était biographique, les enquêtés étaient interrogés sur leur formation, leur parcours professionnel et syndical et sur leurs projets. Puis les questions portaient sur le contenu du travail syndical européen ; les syndicalistes étaient invités à raconter le déroulement de leurs journées de travail, à décrire avec précision leurs différentes tâches syndicales et à recenser les dif-

1. La CGT (Confédération générale du travail), affiliée jusqu'en 1995 à la Fédération syndicale mondiale d'inspiration communiste, entre dans la CES en 1999. La CFDT (Confédération française démocratique du travail), qui résulte de la déconfessionnalisation de la CFTC en 1964, est membre de la CES depuis 1974. La CGT-FO (Force ouvrière), affiliée depuis l'origine à la Confédération internationale des syndicats libres, fait partie des membres fondateurs de la CES. 
ficultés éventuellement rencontrées. La conduite de l'entretien était volontairement peu directive. Mes interventions avaient comme principale fonction de relancer l'enquêté et surtout de le recentrer sur les données objectives de la trajectoire et sur le récit prosaïque des activités quotidiennes concrètes. Ces entretiens ont donc un statut particulier. Les enquêtés se considèrent comme des porte-parole de leur organisation, dont ils donnent la position. Dans le même temps, les discours recueillis lors d'un entretien en face à face ne sont jamais complètement assimilables à une résolution officielle: les digressions sont fréquentes, l'ordre des thèmes abordés et leur importance sont variables. Le locuteur, invité à parler de lui-même, donne inévitablement une coloration propre au discours collectif qu'il met en scène. Ces entretiens font ainsi apparaitre la pluralité des critères de perception et d'appréciation du travail syndical européen.

Tableau 1. Répartition des 31 personnes du corpus en fonction de leur appartenance organisationnelle

\begin{tabular}{lccc}
\hline CES et annexes (8) & $\begin{array}{c}\text { Confédérations } \\
\text { françaises (9) }\end{array}$ & $\begin{array}{c}\text { Fédérations } \\
\text { françaises (7) }\end{array}$ & $\begin{array}{c}\text { Délégués aux comités } \\
\text { d'entreprises européens (7) }\end{array}$ \\
\hline CES (3) & 4 CGT & 2 CGT & 3 CGT \\
$\begin{array}{l}\text { Institut syndical } \\
\text { européen (1) }\end{array}$ & 3 CFDT & 2 CFDT & 2 CFDT \\
$\begin{array}{l}\text { Fédérations } \\
\text { européennes } \\
\text { (4) }\end{array}$ & 2 FO & 3 FO & 2 FO \\
\hline
\end{tabular}

Pour restituer plus systématiquement les principes de cette diversité, nous avons soumis une partie importante des textes constituant le corpus à une analyse de données textuelles lexicales à l'aide du logiciel Alceste, élaboré par Max Reinert (Reinert, 1993)².

L'analyse a été menée sur trente-et-un entretiens (144253 mots). Dans l'ensemble du texte soumis à l'analyse, 8730 formes lexicales distinctes ont été identifiées et parmi elles 4545 formes réduites ont été retenues comme variables actives de l'analyse. Il s'agit de mots réduits à leur radical, de formes conjuguées de verbes ramenées à l'infinitif ou de substantifs accordés ou non en genre ou en nombre (par exemple les mots militer, militant, militantes, militants, militait et milite ont été regroupés et constituent une même forme réduite).

L'analyse menée à l'aide du logiciel Alceste vise à évaluer l'hétérogénéité des phrases qui composent ces entretiens à partir d'une classification hiérarchique descendante. L'objectif n'est pas de comparer des distributions

2. Ce traitement a été effectué avec l'aide de Claude Didry que je remercie vivement. 
statistiques de mots, mais d'étudier la structure formelle de leurs cooccurrences dans les discours. L'ensemble du corpus est découpé en énoncésphrases, repérés par leurponctuation et par les espacements. Cette démarche, qui permet de repérer des associations de mots et de concepts dans des mêmes unités sémantiques et de repérer quels sont les mots qui ne sont pas ou qui sont rarement associés dans une même proposition, conduit à dégager des champs lexicaux distincts.

Le logiciel Alceste fait apparaitre des classes qui regroupent des phrases dont le degré de similarité est proche, c'est-à-dire dont le vocabulaire est proche. Ainsi la phrase «Dans la maison, il y a autant de gens qui viennent de l'appareil militant que de gens qui sont recrutés sur compétences, à la sortie de l'école» est regroupée dans la même classe que la phrase «Ce ne sont pas des gens qui viennent du terrain, des militants: ce sont des gens qui sont recrutés comme une embauche dans une entreprise ». La proximité se traduit par le fait que ces deux phrases partagent un grand nombre de mots: gens, recruter, militant, venir (classe 1 ). En revanche la phrase «Dans le cadre du protocole social, on a réussi à signer trois accords » se trouve dans une classe différente (classe 2). La dissimilarité de cette phrase avec les précédentes est maximale puisqu'elles n'ont pas de mots en commun; elle apparait dès la première partition du corpus en deux grandes classes par le logiciel. Les classes de phrases se définissent par leur profil lexical constitué par les mots les plus fortement associés à chacune des classes.

Ce traitement a ses limites: le regroupement de formes sous leur racine est souvent approximatif; le découpage des unités de contexte est quelquefois discutable; seul le cœur stable des classes est retenu, ce qui conduit à abandonner une partie parfois importante de l'information (Brigidou, Labbé, 2000). Mais le grand intérêt de cette analyse inductive est de ne pas postuler a priori la partition du texte. Ainsi l'hypothèse de départ, selon laquelle les discours des syndicalistes sur l'Europe pouvaient être analysés à partir de l'opposition entre un registre militant qui serait prédominant sur certains thèmes et un discours expert qui prévaudrait sur d'autres thèmes, ne s'est pas révélée pertinente. L'analyse des oppositions qui structurent les discours conduit à reformuler la question du rapport entre expertise et syndicalisme.

\section{L'organisation des discours : les mondes lexicaux des syndicalistes européens}

La partition retenue classe les phrases du corpus suivant un processus qu'on peut décrire par un arbre. Il se dégage de cet arbre deux pôles principaux et cinq classes. Le premier pôle est constitué de mots renvoyant à l'univers des personnes, le second de mots appartenant à l'univers des activités syndicales. 
Au sein du premier pôle, une première subdivision distingue la classe 1 qui porte sur le recrutement des syndicalistes européens, et la classe 4 qui traite de la communication linguistique entre syndicalistes de différents pays.

Au sein du second pôle, deux sous-corpus puis trois classes sont distingués. Le premier (classe 2 ) regroupe les mots appartenant au champ lexical de l'expertise essentiellement économique. Le deuxième regroupe les énoncés ayant trait aux négociations, dans le cadre des comités d'entreprises européens (classe 3) ou du dialogue social européen (classe 5).

Figure 1. Arbre de classification des phrases analysées

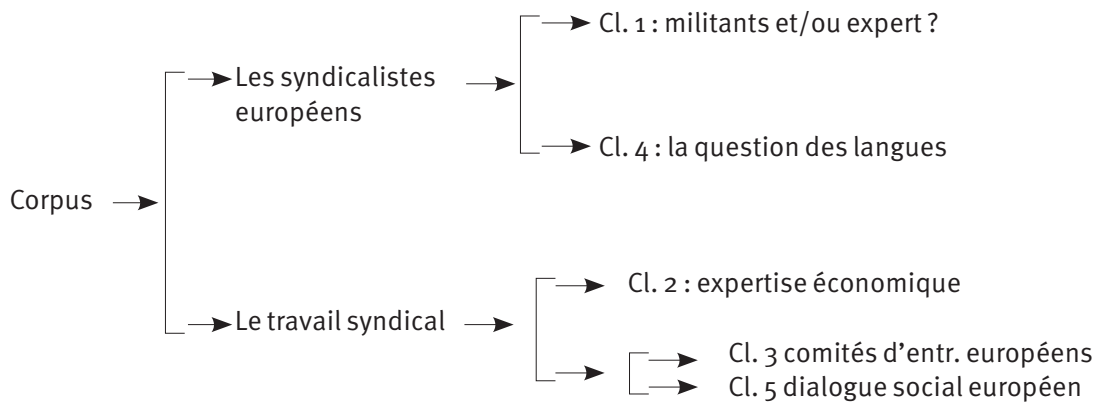

\section{Comment être militant et expert dans un milieu international?}

La première classe et la plus importante regroupe $29 \%$ des énoncés retenus pour l'analyse. Elle est fortement associée aux mots international, militer, rester, gens. Elle regroupe les phrases qui portent sur le milieu des syndicalistes internationaux.

Les termes de cette classe évoquent le fonctionnement particulier de cet univers social. Il s'agit d'un petit univers, qui fonctionne sur le mode de l'interconnaissance et des relations personnelles: gens, milieu, connaitre, humain, rester sont des mots spécifiques de cette classe. Le travail européen suppose des investissements personnels et familiaux importants. On trouve dans cette classe une série de mots appartenant au registre lexical de la famille (femme, père, enfant) ou qui évoquent les contraintes s'exerçant sur le mode de vie des syndicalistes européens (week-end, train, vie, voyage, habiter, partir). Les verbes avoir et être sont très présents, ainsi que toutes les marques de la personne.

Cette classe comprend de nombreux développements sur le mode de recrutement des syndicalistes européens. Les mots sortir, issus, recruter, profil, choisi, parcours font partie du vocabulaire spécifique de cette classe. 
Le recensement du lexique prédominant met bien en évidence le balancement entre deux types d'exigences en partie contradictoires. On trouve, d'une part, le lexique qui renvoie au militant dont la légitimité est d'abord politique : élu, terrain, politique, militantisme, appareil, mandat, interne. Un bon syndicaliste européen représente son organisation à l'étranger. Il est d'abord un militant fiable, qui a une longue expérience de l'intérieur de l'organisation. Mais d'autre part, tout aussi associé à cette classe, on trouve le vocabulaire qui désigne les compétences de type universitaire requises par le travail européen : compétent, école, technicien, expert, connaissance, diplôme, juriste, éducation, université. Comment concilier ces deux exigences? Tel est le thème souvent abordé dans cette première classe: l'examen plus précis par Alceste des modes d'association des mots au sein des phrases montre en effet que les mots militer et compétent sont très fortement associés. Cette association prend des sens contrastés. L'expertise européenne désigne ainsi les compétences spécifiques que doivent développer les militants chargés des questions européennes. Mais cette exigence est aussi au fondement du recrutement de "techniciens», ces jeunes diplômés sans expérience syndicale (et souvent non syndiqués) embauchés pour leur connaissance des dossiers européens. Experts et militants sont alors opposés comme deux populations dont la légitimité repose sur des fondements différents.

Les mots outils les plus spécifiques de la classe 1 sont les verbes de modalité (falloir, pouvoir, savoir), les expressions engageant le point de vue du locuteur (je pense, je crois), ou marquant le degré de certitude (c'est vrai, sans doute, tout à fait). On est ici dans le registre des convictions et des prises de positions subjectives.

\section{La question des langues}

Au sein du premier pôle, une plus petite classe, qui regroupe 13,8 \% des énoncés analysés, a trait à la question des langues. Les mots les plus spécifiques de cette classe 4 sont allemand, anglais, belge, espagnol, français, italien, scandinave. On y trouve des mots relevant du champ lexical de la communication linguistique (parler, langue, communiquer), et plus précisément entre locuteurs de langues différentes (traduction, interprète). De nombreux termes évoquent le niveau en langue (débrouiller, couramment, facile, comprendre, bilingue, maternelle, exprimer) et les apprentissages (cours, apprendre, effort, études, stage). C'est là qu'on trouve le plus d'adjectifs et d'adverbes mesurant l'intensité. Les mots outils spécifiques de cette classe évoquent à la fois le niveau au sens scolaire (bon, fort, bien, mieux, aussi bien) et les liens entre les personnes (avec, entre, proche, vers).

Cette classe a une partie de son vocabulaire en commun avec la première classe sur les compétences syndicales européennes. C'est en effet dans le 
domaine de la connaissance des langues étrangères que les définitions exclusivement militantes de ces compétences marquent le plus immédiatement leurs limites. Les mots problème et handicap font partie du vocabulaire spécifique de cette classe. La maitrise des langues, ou tout au moins de l'anglais (anglais est le mot le plus fortement associé à cette classe) devient une des conditions pour accéder aux lieux du syndicalisme européen. Les enquêtés évoquent ici les obstacles très concrets à la construction de l'Europe syndicale.

La question de l'âge est fortement associée à cette classe avec les mots âge, génération, vieux. Les syndicalistes expriment souvent l'espoir que les nouvelles générations, plus instruites, concilieront plus aisément la maitrise des langues et l'expérience militante.

On trouve enfin dans cette classe des mots qui évoquent les solidarités militantes (camarade, copain). À noter que le mot immigré est associé à cette classe: le recours aux militants immigrés comme traducteurs improvisés, voire comme représentants dans les instances européennes, est apparu comme un des moyens pour concilier les différentes exigences associées au travail européen.

\section{Expertise et négociations sur les questions économiques}

Au sein du second pôle, qui regroupe les énoncés décrivant le contenu du travail syndical, la classe la plus importante regroupe $21,3 \%$ des phrases analysées. Les mots les plus associés à cette classe 2 appartiennent au vocabulaire des analyses macroéconomiques: productif, inflation, salaire, économie, chômage, concurrence, libre, consommation, statistique, théorie, macro, synthèse.

Les phrases de cette classe comprennent ensuite un grand nombre de mots qui évoquent les accords signés lors des négociations au niveau européen: accord, social, signer, partiel (accord sur le temps partiel), parental (accord sur le congé parental), cadre (les accords cadres), convention, textes, droit. Commission (européenne) fait partie des mots fortement associés à cette classe, ainsi que patrons.

Cette classe se caractérise par la prédominance du vocabulaire économique. Mais son lexique est moins uniforme qu'on pourrait le penser. Les termes conflits et grève font aussi partie de son vocabulaire spécifique. Les mots outils associés à cette classe renvoient à des négations ou à des jugements négatifs (mal, point, pas), à une opposition (contre, en face). Cette classe comprend un nombre significativement important de verbes de modalités évoquant les convictions et les positions de l'interlocuteur: croire, vouloir. Elle est donc plus personnalisée et plus conflictuelle que ne pourrait le laisser à penser la prédominance du vocabulaire expert, au sens cette fois où l'expertise désigne un champ spécialisé de connaissances académiques. 


\section{Les comités d'entreprise européens}

La classe 3 ( $23 \%$ des énoncés analysés) regroupe les considérations sur les comités d'entreprises européens. Ces instances, conçues pour faire «descendre » le dialogue social au sein des entreprises multinationales, font l'objet d'une grande attention de la part des différents observateurs institutionnels européens. Les mots associés à cette classe évoquent l'organisation des comités européens (réunir, délégué, plénière, fonctionnement, liaison, représentant, budget, siéger, forum) et leurs prérogatives (information, consultation, expert).

Entreprise est le mot le plus spécifique de cette classe, évoquée aussi sous les termes société, filiale, site, usine, multinationale ou à partir d'exemples (dans l'ordre:Danone, Elf, Thomson, Michelin). Direction (et non plus patronat) est un mot spécifique de cette classe, ainsi que salariés. Plusieurs termes évoquent les politiques d'entreprises qui menacent l'emploi (licenciement, restructuration).

Dans cette classe, on trouve le vocabulaire des revendications (demander, revendiquer) et des luttes, notamment sur le terrain judiciaire (avocat, appel). C'est cette classe qui contient le plus de verbes et où prédomine le vocabulaire de l'action (décider, aller, avancer). Mais le vocabulaire renvoyant aux expertises est également spécifique de cette classe : expert, séminaire, expertiser. L'expertise désigne cette fois une prérogative des comités d'entreprise européens qui peuvent demander à bénéficier des services d'un expert de leur choix.

\section{Les dimensions institutionnelles du dialogue social}

Une petite classe (12,4\% des énoncés) évoque le dialogue social avec le patronat européen, non plus sous l'angle du contenu des accords conclus, mais sous son angle institutionnel. Elle comprend des expressions appartenant au registre des organisations et de leurs rouages (comité exécutif, congrès, instance, syndicat, confédération, fédération, titulaire, suppléant, groupe de travail). On y trouve les développements sur le dialogue de branche (branche, sectoriel, chimie, agriculture, mine), sur le travail de réunion (préparer, prochain, décembre, actualité) et sur les dimensions les plus organisationnelles de ce travail (assister, voter, siège, réunion). Le patronat est désormais évoqué par le sigle de l'Union des confédérations de l'industrie et des employeurs d'Europe (Unice). Le sigle CES est très fortement associé à cette classe.

C'est la classe la plus impersonnelle, où le locuteur s'implique le moins en tant que personne. 


\section{La logique des prises de position}

Comment se distribuent ces différentes classes? Chaque entretien se caractérise par une proximité plus ou moins importante à l'égard d'un ensemble de phrases significatives au sein de chaque classe. Les entretiens sont différenciés dans l'analyse par des variables supplémentaires: le type de structure syndicale de l'interviewé (une structure européenne, une confédération nationale, une fédération, un comité d'entreprise européen), le nom de la centrale syndicale (CGT, CFDT ou FO).

Il a également été tenu compte du type de parcours du syndiqué: on a distingué les enquêtés qui n'ont pas fait d'études supérieures et qui ont eu un parcours militant (17 enquêtés, essentiellement en France), les experts recrutés sur diplôme et non syndiqués (7 personnes) et les syndicalistes ayant une trajectoire mixte, c'est-à-dire à la fois une formation universitaire et un parcours militant (7 personnes).

Tableau 2. Répartition des 31 personnes du corpus en fonction de leur parcours

\begin{tabular}{lcccc}
\hline & $\begin{array}{c}\text { CES et } \\
\text { annexes } \\
(8)\end{array}$ & $\begin{array}{c}\text { Confédérations } \\
\text { françaises } \\
(9)\end{array}$ & $\begin{array}{c}\text { Fédérations } \\
\text { françaises } \\
(7)\end{array}$ & $\begin{array}{c}\text { Délégués de comités } \\
\text { d'entreprises } \\
\text { européens (7) }\end{array}$ \\
\hline $\begin{array}{l}\text { Parcours } \\
\text { militant, } \\
\text { pas d'études } \\
\text { sup. }\end{array}$ & 1 & 4 & 6 & 6 \\
$\begin{array}{l}\text { Diplômé } \\
\text { non syndiqué }\end{array}$ & 4 & 3 & 0 & 0 \\
$\begin{array}{l}\text { Parcours mixte } \\
\text { (diplômé et } \\
\text { militant) }\end{array}$ & 3 & 2 & 1 & 1 \\
\hline
\end{tabular}

L'analyse détermine, pour ces variables, un degré d'association avec les classes de phrases. La classe 1, qui pose la question de la légitimité politique des experts (ou de la compétence technique des militants), est fortement représentée dans les entretiens auprès des responsables des questions européennes dans les confédérations françaises. Conduits à se situer à la fois par rapport à leur confédération nationale et par rapport aux institutions européennes, ils ressentent avec acuité les contradictions entre les critères nationaux et les critères européens de définition de la légitimité syndicale. La définition militante des compétences syndicales se trouve confrontée à la tendance du syndicalisme européen à recruter, en dehors du champ syndical, 
des experts diplômés. Cette pratique se met en place dans les confédérations syndicales françaises, qui sont donc des milieux de travail où ces deux profils de recrutement et de définition du syndicalisme sont directement confrontés.

La classe 4, qui porte sur la question des langues, est très présente chez les syndicalistes non diplômés ayant eu un parcours militant: ce sont eux qui subissent les problèmes posés par l'imposition croissante de l'anglais dans les négociations européennes. Une partie importante des phrases de cette classe émane de syndicalistes travaillant dans une fédération de syndicat français. À ce niveau de l'activité syndicale, les problèmes de communication linguistiques apparaissent en effet particulièrement aigus. Dans les fédérations, contrairement à ce qui se passe à la CES ou dans les départements Europe des confédérations, les syndicalistes chargés des questions européennes ne parlent pas toujours les langues étrangères. Or, moins riches que la CES, les fédérations européennes ont aussi moins de moyens pour assurer le travail de traduction et d'interprétariat. De nombreuses réunions se tiennent uniquement en anglais et le nombre de documents systématiquement traduits est restreint. Le niveau fédéral apparait ainsi comme un lieu où se nouent un certain nombre de tensions et d'obstacles pratiques à l'internationalisation du syndicalisme. On touche peut-être là une des limites du dialogue social sectoriel que cherchent à développer les instances européennes (Dufresne, 2002).

Comment se distribuent les discours sur les différents aspects du travail syndical européen (second pôle)? On trouve là une nette distinction entre les développements sur le travail dans les institutions européennes, d'une part (les classes 2 et 5), et dans les entreprises d'autre part (classe 3). Cette distinction reproduit d'abord logiquement celle des niveaux d'activité syndicale. Les syndicalistes travaillant à Bruxelles, à la CES ou dans un institut périphérique contribuent significativement aux énoncés de la classe 2 sur l'expertise économique et de la classe 5 sur les institutions, alors que le thème des comités d'entreprises est plus spécifiquement développé par les syndicalistes siégeant dans les entreprises.

Le type de trajectoire de l'enquêté, ensuite, intervient pour rendre compte du discours développé. Les locuteurs recrutés "sur compétences» en dehors du champ syndical, les non-syndiqués, sont fortement associés au discours sur l'expertise économique et sur le dialogue social européen. Inversement, le thème de l'entreprise est abordé plus souvent par les enquêtés qui ont eu un parcours militant.

Enfin, le syndicat de l'enquêté doit aussi être pris en compte. La CFDT (bien représentée dans les instances européennes au moment de l'enquête) occupe une place importante dans la production des énoncés de la classe 5 sur les institutions, alors que la CGT et FO contribuent plus nettement aux énoncés de la classe sur les comités d'entreprises. 
L'analyse factorielle des correspondances qui aboutit à la projection des cinq classes et des variables supplémentaires sur un plan permet de synthétiser ces résultats3.

\section{Figure 2}

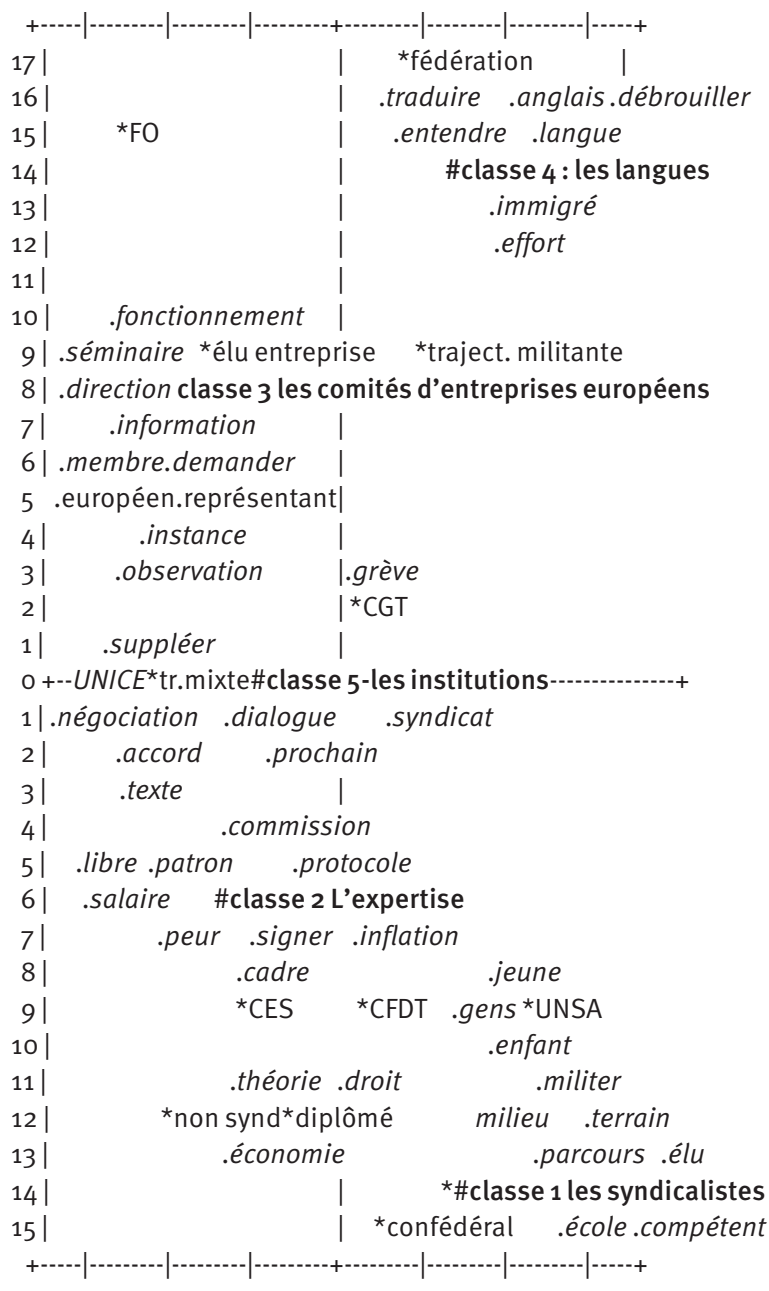

Sur l'axe horizontal (axe 1) se séparent les deux principaux pôles de la classification opérée par Alceste: on trouve à droite du graphique les classes sur le milieu des syndicalistes, à gauche celle sur les activités syndicales. L'axe vertical (axe 2) oppose deux manières de se représenter le syndicalisme

3. Cette analyse est réalisée à partir d'un tableau croisant les classes et les lexèmes. L'axe 1 porte $32 \%$ de l'inertie, l'axe $225 \%$. Le schéma reproduit ici est simplifié. 
européen : d'une part (en haut du graphique) on trouve des représentations plus concrètes du monde du travail et de la mise en pratique du syndicalisme européen (l'entreprise, les langues), de l'autre (sous l'abscisse) les considérations plus théoriques sur l'économie, le droit et les institutions européennes. Cet axe sépare assez nettement les enquêtés en fonction de leurs parcours, distinguant les discours des syndiqués ayant eu un parcours militant (en haut), de ceux qui sont recrutés en fonction de leur diplôme (en bas).

Cette analyse lexicale conduit à repenser les modes de différenciation entre expertise et militantisme par rapport aux hypothèses initiales. Trois grands résultats peuvent en effet être dégagés.

En premier lieu, les énoncés regroupent le plus souvent à la fois des références à l'expertise et au militantisme. Ces deux dimensions sont centrales dans les préoccupations des syndicalistes chargés des questions européennes. C'est même la confrontation et la tension entre ces deux registres qui définit la classe 1, la plus importante du corpus. Dans les discours sur le travail syndical européen, le lexique de l'expertise domine, mais le vocabulaire des revendications et du conflit reste présent, surtout dans les énoncés sur les comités d'entreprises européens. Seule la petite classe 5 , sur les dimensions institutionnelles du dialogue social européen, ne comprend aucun mot évoquant l'action ou la revendication syndicales. La projection des mots analysés sur le plan confirme la position relativement centrale de mots comme grève ou militer, qui se trouvent associés à différentes classes d'énoncés.

En second lieu, la distinction entre militantisme et expertise s'avère importante pour rendre compte de l'organisation du corpus, si l'on se penche sur les trajectoires des locuteurs. Les enquêtés qui se définissent comme des militants ne développent pas les mêmes thèmes et n'utilisent pas le même lexique que les experts syndicaux recrutés sur compétences. Les seconds adoptent plus systématiquement des discours théoriques avec un large usage du registre de l'institutionnel européen. Les diplômés parlent du fonctionnement des institutions européennes, de la construction des expertises syndicales et des compétences syndicales européennes. Les militants font plus systématiquement référence à l'action syndicale et au militantisme. Ils abordent plus souvent la question des obstacles concrets, notamment linguistiques, à la construction du syndicalisme européen. Ils portent aussi plus d'intérêt aux effets pratiques, sur les rapports sociaux au sein des entreprises, de l'instauration de ce syndicalisme européen. Du point de vue des discours produits, l'opposition entre les diplômés et les militants recoupe celle entre les experts travaillant à Bruxelles ou dans une confédération, d'une part, et les syndicalistes des fédérations ou des comités d'entreprise européens, d'autre part.

Cette division du travail dans la production de discours sur le syndicalisme européen traduit en partie la division du travail entre les syndicalistes des différents niveaux. On retrouve ainsi, dans les visions du travail syndical euro- 
péen, l'opposition entre les catégories des «syndicalistes d'en haut» proches des institutions européennes, d'une part, et des «syndicalistes d'en bas» plus proches des militants et salariés d'entreprises, d'autre part. Cette division n'est pas exempte de tensions et de contradictions, comme l'attestent les modes d'imbrication du vocabulaire de l'expertise et du militantisme dans les différentes classes.

En effet, et c'est là le troisième enseignement de cette analyse, l'expertise et le militantisme sont associés de manières contrastées dans le corpus. Ils sont opposés et pensés comme contradictoires dans les énoncés qui portent sur les modes de légitimation du syndicaliste européen. Experts et militants constituent alors deux populations sélectionnées selon des principes différents. Mais dans les phrases du second pôle sur le travail syndical européen, et notamment dans les phrases portant sur les comités d'entreprise européens, l'expertise est associée au lexique militant dans un autre sens: le droit à bénéficier d'une expertise fait cette fois partie des revendications syndicales, l'expertise y compris européenne est cette fois considérée comme une ressource que les militants cherchent à mobiliser dans les rapports de force au sein de leur entreprise.

Si le syndicalisme européen a indéniablement produit ses experts et ses professionnels de l'Europe, il s'enracine dans des milieux sociaux et institutionnels plus hétérogènes que d'autres lieux de production d'une expertise européenne. Le rapport à l'expertise est alors aussi plus conflictuel, comme l'atteste bien la diversité des manières de poser cette question en fonction des positions et des trajectoires des acteurs syndicaux.

\section{Références}

Brugidou Mathieu, LABBe Dominique, 2000, Le discours syndical français contemporain (CFDT, CGT, FO en 1996-1998), Grenoble, CERAT, p. 13.

DUFRESNE Anne, 2002, "La branche, niveau stratégique dans la coordination des négociations collectives? ", Chronique internationale de l'IRES, $\mathrm{n}^{\circ} 74$, janvier.

GobIN Corinne, 1993, «La CES. Un vocabulaire syndical européen », Mots. Les langages du politique, $\mathrm{n}^{\circ} 36$.

- 1997, L'Europe syndicale: entre désir et réalité, Bruxelles, Labor.

- 2003, «De l'international au mondial: la CES aux prises avec la mondialisation», Mots. Les langages du politique, $\mathrm{n}^{\circ} 71$.

PERnot Jean-Marie, 1998, «Une université européenne du syndicalisme? L’Europe des syndicats », Politix, $\mathrm{n}^{\circ} 43$.

REINERT Max, 1993, "Les mondes lexicaux et leur logique à travers l'analyse statistique d'un corpus de récits de cauchemars ", Langage et société, n64, p. 5-39.

WAGNER Anne-Catherine, 2005, Vers une Europe des syndicats. Une enquête sur la CES, Paris, Le Croquant (Savoir agir). 